\title{
Black Triangle, Etiology and Treatment Approaches: Literature Review
}

\author{
Putri Masraini Lubis \\ Resident \\ Department of Periodontology \\ Faculty of Dentistry, University of Sumatera Utara \\ putrimasraini@gmail.com
}

\author{
Rini Octavia Nasution \\ Lecturer \\ Department of Periodontology \\ Faculty of Dentistry, University of Sumatera Utara
}

\author{
Zulkarnain \\ Lecturer \\ Department of Periodontology \\ Faculty of Dentistry, University of Sumatera Utara
}

\begin{abstract}
Currently, beauty and physical appearance is of a major concern for many people, along with the greater demands of aesthetics in the field of dentistry. Aesthetics of the gingival is one of the most important factors in the success of restorative dental care. The loss of the interdental papillae results in a condition known as the black triangle. Interdental papilla is one of the most important factors that clinicians should pay attention to, especially in terms of aesthetic. The Black triangle can cause major complaints by the patients such as: aesthetic problems, phonetic problems, food impaction, oral hygiene maintenance problems. The etiology of black triangle is multi factorial, including loss of periodontal ligaments due to recession, reduced alveolar bone height associated with interproximal contact, length of embrasure area, root angulation, position of interproximal contact, triangular crown, aging, and midline diastema. Some of the handling treatment includes non-surgical and surgical methods. Non-surgical treatments include ceramic veneer or crown, addition of composites for interdental papilla formation, apical bracket installation and use of gingival prosthesis, while surgery includes recontouring, preservation and reconstruction of the interdental papilla. This article will discuss the definition, etiology, classification and various considerations in handling the case of black triangle.
\end{abstract}

Keywords-black triangle, interdental papilla, papilla reconstruction

\section{INTRODUCTION}

The current awareness of beauty level has driven has driven a higher demand in aesthetic dentistry. The need for cosmetic dentistry to improve appearance in recent years is increasing. Cosmetic dental procedures and periodontal treatment has become inseparable. Successful dental aesthetic care helps to restore the patient's self-image, social skills and gain professional success experience. In the past, periodontal treatment was more directed at preservation care and periodontal health restorations than aesthetic appearances. However, the demand for aesthetics has increased the ability of periodontist to overcome aesthetic problems in patients [1].
Loss of the interdental papillae results in a condition known as the black triangle. Various factors may affect in the case of interdental papilla loss, including alveolar crest height, interproximal spacing, soft tissue, buccal thickness, and extent of contact areas. With the current adult population which mostly has periodontal abnormalities, open gingival embrasures are a common thing. Open gingival embrasures also known as black triangles occur in more than one-third of the adult population; black triangle is a state of disappearance of the interdental papillae and is a disorder that should be discussed first with the patient before starting treatment. One of the greatest aesthetic difficulties in periodontal plastic surgery relates to the ability to rebuild the missing papilla on the anterior portion of maxilla [2].

The interdental papilla is part of the gingiva that fills the space between two teeth. Not only serves as a biological barrier for the periodontal structures underneath, it also has an important role in aesthetics. Common causes of interdental papilla loss are midline diastema, branched root, tooth extraction, oral traumatic interproximal oral procedures, abnormal crown form and periodontal disease [2]. Several non-surgical and surgical measures have been suggested to treat soft tissue deformities and treat interproximal rooms. Nonsurgical measures that can be performed in interdental papilla regeneration include restorative action, orthodontic treatment, prosthetic treatment and repetitive curettage in papillae. While surgical measures include papilla recontouring, papilla preservation, papilla reconstruction. Surgical techniques for papilla reconstruction include pedicle graft, semilunar coronally repositioned papilla, and envelope-type flap. In order to enforce the diagnosis for treatment of cases of black triangle, the etiological factor should be eliminated before determining the treatment [3].

\section{LITERATURE REVIEW}

A. Definition

Interdental papilla is part of the gingiva that fills the space between two teeth [2]. The loss of interdental 
papilla results in a state known as the black triangle (Figure 1). The interdental papilla not only serves as the biological barrier for the periodontal structures beneath it, but also has an important role in aesthetics. The interdental papilla is formed from a dense connective tissue, and is limited by the contact between the teeth, the width of the proximal tooth surface and the cementum enamel junction (CEJ). Black triangles are more common in adults who underwent orthodontic treatment $(38 \%)$ than in adolescents who also underwent orthodontic treatment (15\%). However, $41.9 \%$ of adolescent patients who had performed orthodontic treatment due to maxillary anterior crowding cases generally had an anterior case of open gingival embrasure [2].

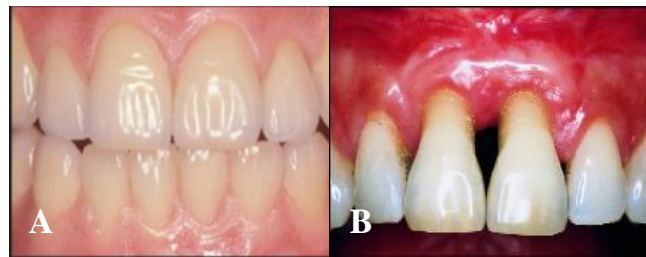

Figure 1. A. Normal interdental papilla B. Maxillary black triangle.

\section{B. Etiological factors of black triangle}

The etiology of black triangle is a multifactorial (Figure 2). Cause of black triangle includes loss of periodontal ligaments due to recessions, reduced alveolar bone height associated with interproximal contact, length of embrasure area, angulated root, and position of interproximal contact, triangular crown, aging, and midline diastema [2,3,5]. It is important to note that, predisposing etiological factors leads to the occurrence of black triangles in addition to common biological factors. Changes in papilla dimension during orthodontic alignment can be seen due to treatment of periodontal disease, tooth extraction and iatrogenic accident treatment such as veneer and unsuitable crown.

Existence of a black triangle can be related to the age factor. Research from Ko-Kimura and colleagues showed that patients over 20 were more likely to have a black triangle than those less than 20 years of age. The percentage of black triangle was found to be $67 \%$ in population over 20 years and $18 \%$ in population under 20 years [2]. This is due to depletion of oral epithelium, reduced keratinization of gingiva and reduction of papilla height due to aging process [2].

Black triangle can cause patient complaints such as aesthetic problems, phonetic problems, food impaction, and oral hygiene maintenance problems.

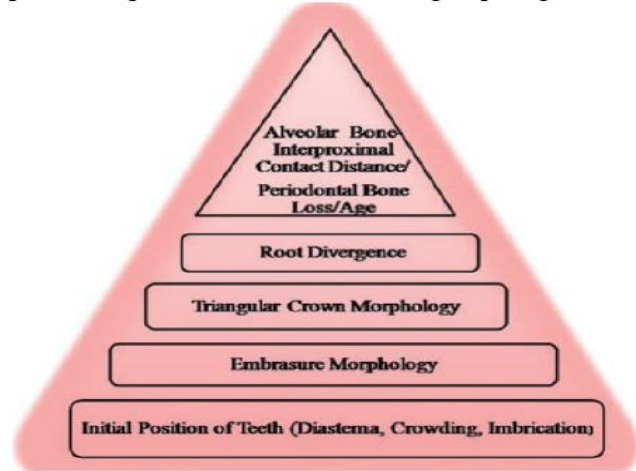

Figure 2. Hierarchy of the etiological factor of the black triangle.

\section{Classification of black triangle}

The loss of the interdental papilla is classified by Nordland and Tarnow. This classification is based on three anatomical signs: the interdental contact point, the most coronal point of cemento enamel junction (CEJ) on the interproximal surface and the most apical point of the CEJ on the labial surface.

Four classes were identified (Figure 3):

- Normal: the interdental papilla fills the niche up to the apical extension of the interdental contact point

- Class I: the tip of interdental papilla is placed between interdental contact point and the most coronal point of CEJ at interproximal surface.

- Class II: the tip of papilla is placed between the most coronal point of ECJ at interproximal surface and the most apical point of CEJ at labial surface.

- Class III: the tip of the interdental papilla is at the ECJ or it is apically to the most apical point of CEJ at the labial surface.

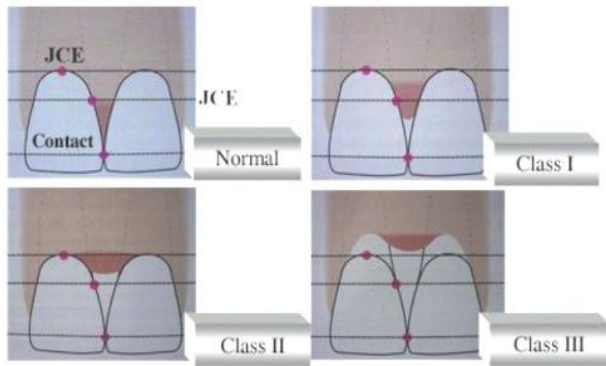

Figure 3. Classification of black triangle.

\section{Management of cases of black triangle}

- Non-surgical approach

In the case of the occurrence of black triangle caused by trauma while brushing, hygiene in the interdental area should be modified; the toothbrush must be replaced, allowing for re-epithelialization of traumatic injury that can restore papilla $[2,4]$.

\section{Restorative approach}

For treatment of black triangle through restorative considerations, it should be noted that to change the position of the point of contact, one of them with ceramic veneer or crown. If possible add pink porcelain to the restoration to manipulate the presence of interdental papilla loss [2]. The advantages of such methods are biocompatibility of the material, stable 
color and non-porous surface, preventing better plaque attachment than composite resins. The disadvantages are skill and hard to fix [4].

In addition, restoration of cervical mesial regions will reduce the presence of gingiva by changing coronal shape. Composite can be inserted near gingival sulcus as a guide for the formation of an interdental papilla [2]. The advantage is that the composite has many colors that are stable and wear resistant; the latest generation of dental bonding agents enables the bonding of composites to dentine. The disadvantage is that there may be changes in bonding, discoloration, fluid seepage through the dental interface and composite [4].

Another method which can be used such as interproximal enamel reduction, using diamond strips to reshape the mesial surface of the upper central incisors. Approximately 0.5 to $0.75 \mathrm{~mm}$ of enamel is reduced in the interproximal region, which increases the point of contact and decreases the gingiva. Decreased interproximal enamel on teeth with triangular crowns will change the point of contact on a large area thus forming a gingival embrasure [2].

\section{Orthodontic approach}

Orthodontic treatment is aimed at reducing black triangle space and is done by placing more contact points into the apical region (Figure 4), so that the height of the alveolar bone and papilla can be induced by the movement of orthodontic extrusion. Divergent roots are generally associated with black triangle space. Divergent roots can also be caused by the incorrect mounting of brackets, not perpendicular to the axis of the tooth, so it is important to analyze the periapical radiograph prior to the installation of the bracket [2].

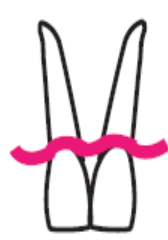

Figure 4. A. Divergent roots B. Orthodontic bracers C. Convergent

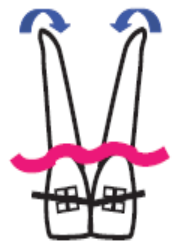

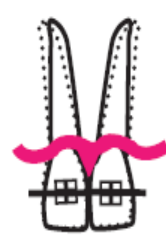
roots.

Interproximal contact will move the point of contact to a larger area, thereby reducing the open gingival embrasure. Gingival embrasures can be caused by the direction of movement of the teeth and the thickness of the labiolingual of the bone and soft tissue, which usually occurs in orthodontic treatment. During the movement of the tooth toward the lingual, the gingival tissue will thicken and move in the occlusal direction of the facial aspect of the tooth. Conversely, the movement of the teeth toward the labial will cause the gingival tissue to become thin and move more apically. Volume of soft tissue in the gingival embrasure region depends on the existing bone, the height of the bone, and the severity of the diastema. Closing the diastema by orthodontic compresses the soft tissues then fills the embrasure chamber (Figure 5) [2].
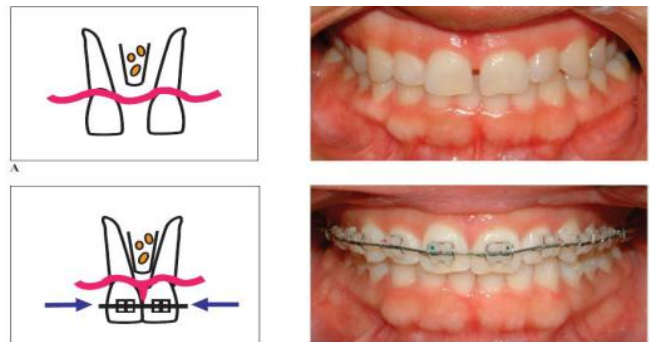

Figure ${ }^{\mathrm{B}}$ 5. Closure of diastema and papilla regeneration. A. Tooth prior to orthodontic treatment indicates the presence of diastema B. closure of the diastema with the formation of an interdental papilla fills an empty space.

\section{Prosthodontics approach}

A very simple but effective procedure for managing a good gingival recession and loss of interdental papillae is the use of gingival prosthesis. Gingival epithesis is a removable mask or aesthetic and functional prosthesis covering the missing gingival tissue [4]. The indication is defective in interdental with a gap between the contact point and alveolar crest> 5 $\mathrm{mm}$, in patients unable to undergo repeated surgical procedures. Contra indications: patients with poor and unstable periodontal health, poor oral hygiene, patients with high caries risk. Advantages: Noninvasive, easy maintenance, splinting on the teeth can be done, more economical. Disadvantages: required patient's cooperation, food impaction and place of bacteria growth, possibly can damage or change the color of prosthesis. Various materials that can be used are: Auto and heat polymerizing acrylic resin, rigid, flexible material, copolyamide, soft silicone material [4].

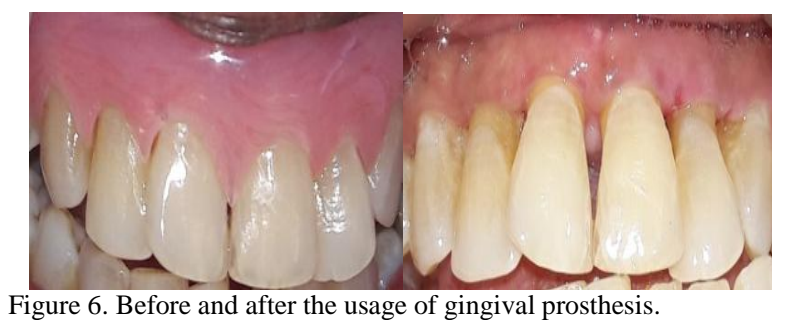

Figure 6. Before and after the usage of gingival prosthesis.

\section{- Surgical approach}

To support the success of surgical treatment required thick gingival biotype characteristics and no loss of periodontal ligament. Patients with a thin gingival biotype are susceptible to recessions that are also susceptible to the occurrence of black triangles. This is due to thicker gingival biotypes having better vascularization that facilitates the healing process [2]. Surgical techniques aim to reshape, maintain, or repair soft tissue between teeth with implant [1].

Surgical approaches include:

1. Papilla recounting to reshape soft-tissue contours.

2. Papilla preservation to reduce and prevent replacement of the gingival margins more apical after surgery, this technique developed by Takei et al and Cortelini et al.

3. Papilla reconstruction after inflammation is removed; the technique is a combination of pedicle flap and papilla preservation $[2,4]$. 
Surgical techniques may be used pedicle flaps, free gingival and sub epithelial connective tissue graft. Some case reports have demonstrated success with sub epithelial connective tissue graft and orthodontic therapy. According to Wu et al., flap surgery has shown better results than free gingival graft. Grupe et al. stated that the techniques with pedicle flaps showed better results than free gingival graft techniques, because the blood supply is provided by the base of the pedicle [2].

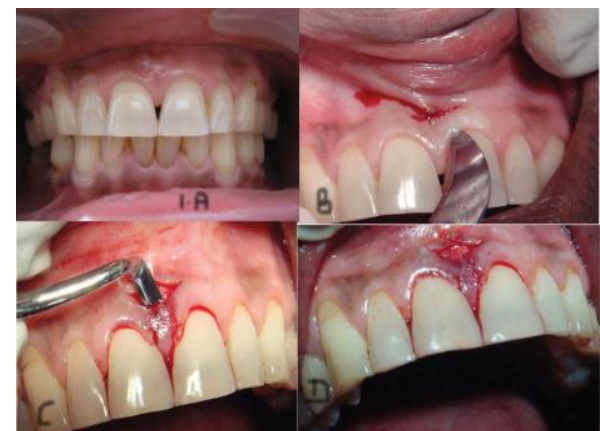

Figure 7. A. Pre-operative (presence of "black triangle" between maxillary central incisors), B. Crevicular incision followed by semilunar incision, $\mathrm{C}$. Coronal displacement of gingiva papillary, D. Void created by displacement of gingiva papillary.

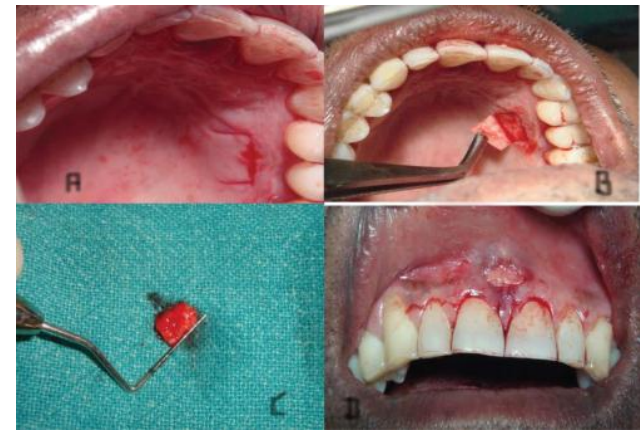

Figure 8. A. Trap door incision on donor site (palate), B. Partial thickness flap elevation, C. Harvested subepithelial connective tissue graft, D. Interposed subepithelial connective tissue graft at the recipient site.

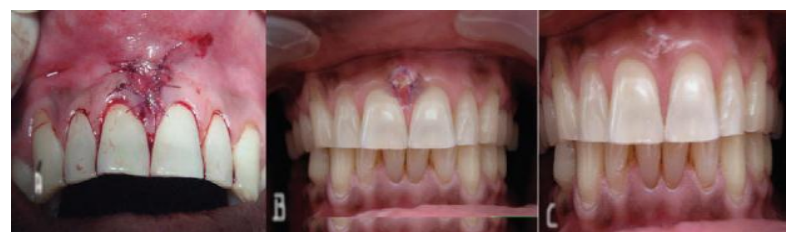

Figure 9. A. Securing subepithelial connective tissue graft with 6-0 vicryl suture, B. Healing after 1 month, C. Healing after 6 months

\section{DISCUSSION}

Loss of the interdental papillae results in a condition known as the black triangle. Interdental papilla is one of the most important factors that clinicians should pay attention to, especially in terms of aesthetic. Various factors may affect in the case of interdental papilla loss, including alveolar crest height, interproximal spacing, soft tissue, buccal thickness, and extent of contact areas.

It is important to observe the vertical distance between the bone crest and the apical point of the intermediate contact area, and the soft tissue height in the interdental area. If the distance between the bone crest and the contact point $\leq 5 \mathrm{~mm}$ and the papilla height $<4 \mathrm{~mm}$, the surgical procedure to raise the volume of the papilla may be performed. If the distance between the bone crest and the contact point is $>5 \mathrm{~mm}$ due to loss of periodontal tissue support, nonsurgical procedures with a combination of restoration procedures may be performed.

Selection of surgical procedures related to reconstruction of the gingival tissue, attention should be given for adequate blood intake. Due to regional limitations to papilla regeneration, any grafting procedure will affect the urgent availability of dirt needed in the papilla reconstruction action. Therefore, the selected surgical technique should be able to provide adequate blood supply from the flap to the graft material, maintain the integrity of the papilla as well as to prevent occurrence of flap necrosis.

The loss of the interdental papillae results in a condition known as the black triangle. Interdental papilla is one of the most important factors that clinicians should pay attention to, especially in terms of aesthetic. A multidisciplinary approach must be considered mandatory if a successful clinical outcome is to be achieved. All etiological factors and treatment alternative must be discussed with the patient before starting the treatment.

\section{REFERENCES}

[1] A. Kaushik, P.K. Pal, K. Jhamb, D. Chopra, V.R. Chaurasia, V.S. Masamatti, "Clinical evaluation of papilla reconstruction using subepithelial connective tissue graft," Journal of Clinical and Diagnostic Research, vol. 8(9), pp. 77-81, 2014.

[2] J.D.D. Oliveira, C.M. Storrer, A.M. Sousa, T.R. Lopes, J.D.S. Vieira, T.M. Deliberado, "Papillary regeneration: anatomical aspects and treatment approaches," RSBO, vol. 9(4), pp. 44856, 2012.

[3] B.K. Al-Zarea, M.G. Sghaireen, W.M. Alomari, H. Bheran, I Taher, "Black triangles causes and management: a review of literature," British Journal of Applied Science \& Technology, vol. 6(1), pp. 1-7, 2015.

[4] Y. Ravishankar, K. Srinivas, S.K. Sharma, S.P. Kumar, "Management of black triangles and gingival recession: a prosthetic approach," Indian Journal of Dental Sciences, vol. 4(1), pp. 141-145, 2012.

[5] P. Palathingal, J. Mahendra, "Treatment of black triangle by using a sub-epithelial connective tissue graft," Journal of Clinical and Diagnostic Research, vol. 5(8), pp.1688-1691, 2011.

[6] M. Agarwal, M. Mittal, S. Mehrotra, A. Agarwal, "Black triangle and its reconstruction: a review," Journal of Dental Sciences \& Oral Rehabilitation, pp. 55-56, 2011.

[7] B. Cohen, "Pathology of the interdental tissues," Dent. Pract., vol. 9, pp. 167-173, 1959.

[8] V.G. Kokich, Adjunctive role of orthodontic therapy. In Carranza's clinical periodontology, $11^{\text {th }}$ ed., 2012, pp. 505-506.

[9] N. Carranza, C. Zogbi, "Reconstruction of interdental papilla with an underlying subepithelial connective tissue graft: technical considerations and a case reports," Int. J. Periodontics Restorative Dent., vol. 31(11), pp. e45-50, 2011.

[10] R.C.N.D.C. Pinto, B.L. Colombini, S.K. Ishikiriama, L. Chambrone, F.E. Pustiglioni, G.A. Romito, "The subepithelial connective tissue pedicle graft combined with the coronally advanced flap for restoring missing papilla: A report of two cases," Quintessence Int., vol. 41(3), pp. 213-220, 2010.

[11] A.A. Sharma, J.H. Park, "Esthetic considerations in interdental papilla: remediation and regeneration," J. Esthet. Restor. Dent., vol. 22, pp. 18-30, 2010. 